\title{
Parametric STUdy OF THE Elementary SegMent OF AN ANTIPRISMATIC POP-UP DEPLOYABLE LATTICE COLUMN
}

\author{
Noémi Friedman', György Farkas², Adnan Ibrahimbegovic ${ }^{3}$ \\ ${ }^{1}$ Institut für Wissenschaftliches Rechnen, Technische Universität Braunschweig, Germany \\ n.friedman@tu-braunschweig.de \\ ${ }^{2}$ Dept. of Structural Engineering, Faculty of Civil Engineering, Budapest Univ. of Technology \\ and Economics \\ farkas@vbt.bme.hu \\ ${ }^{3}$ Sorbonne Universités/UT Compiègne, Centre de recherches Royallie, Compiègne, France \\ adnan.ibrahimbegovic@utc.fr
}

\begin{abstract}
In this article the primary segment of an antiprismatic pop-up mast is analyzed, that can be applied for largely flexible architectural designs, like deployable bridges or transportable look-out towers. This deployable column, consisting of rigid plates, rigid and elastic bars, is characterized by its selfdeploying behavior due to the energy accumulated from lengthening the elastic bars during packing. The main goal of this paper is to prepare the analysis of the complex structure by a herein detailed investigation of the behavior of one, basic element of the deployable mast. After the analytical examination of the general behavior of the basic segment a geometrically nonlinear finite element formulation is used to trace the force-displacement diagram. Besides the parametric study, approximations of main mechanical parameters are herein given for facilitating preliminary design of such deployable structures.
\end{abstract}

Keywords: deployable structure, antiprismatic structure, non-linear analysis, Yoshimura pattern, instability analysis

\section{NOMENCLATURE}

$\begin{array}{ll}- & h \\ - & h^{\varphi} \\ - & R \\ - & R^{\varphi} \\ - & n \\ - & \phi=180^{\circ} / n \\ - & l_{p} \\ - & l_{p}^{\varphi} \\ - & l_{b} \\ - & \lambda=\frac{l_{p}^{\varphi}}{l_{p}} \\ - & \lambda_{\max } \\ - & N \\ - & N_{p}=N / n\end{array}$

\footnotetext{
height of both antiprisms of the basic unit in the reference (initial, deployed) state height of both antiprisms of the basic unit in the actual state radius of the horizontal polygons in the reference (initial, deployed) state radius of the horizontal elastic polygons in the actual state number of the vertices of the horizontal polygons of the antiprism half of the inner angle of the horizontal polygons length of each horizontal bar in the reference (initial, deployed) state length of the elastic horizontal bars in the actual state length of the rigid bracing bars stretching of the elastic horizontal bars during packing maximal stretching of the horizontal bars (in the completely packed state) total packing force applied at the top polygon of the basic unit packing force applied at each vertices of the top $n$-gon of the basic unit
} 


$$
\begin{array}{ll}
- & S_{p} \\
- & \varepsilon \\
- & h_{c r}^{\varphi} \\
- & x_{c r}=\frac{h_{c r}^{\varphi}}{R} \\
- & N_{c r} \\
- & E A
\end{array}
$$

tension force in the elastic horizontal bars during packing (actual state)

strain in the horizontal elastic bars (actual state)

height of both antiprisms of the basic unit in the critical state

relative critical height of the segment

critical packing force (maximum value of $N$ )

(linear) axial stiffness of the elastic bars

\section{INTRODUCTION}

An antiprism is a polyhedron composed of two parallel, identical polygons connected by triangles. Antiprisms are similar to prisms except the two polygons are twisted relative to each other and thus the lateral faces are not quadrilaterals but triangles (Fig. 1). A regular antiprism is formed with a $180 / \mathrm{n}$ degree of twisting angle between the two n-sided polygons. Concerning mechanical behavior of space truss systems generated from the edges of a regular antiprism the results are rather fascinating. Tarnai had shown that a space truss generated from even sided regular antiprism is a finite mechanism while the ones from odd sided antiprisms are stiff forms [1]. Nevertheless, by building stiff truss units (formed from odd sided antiprisms) on top of each other, forming a cylindrical truss, small displacements of boundary joints at the bottom grow exponentially along the height [2]. Consequently it is rather to be avoided to apply such regular antiprismatic trusses for conventional engineering applications without any additional stiffening. Nonetheless, this geometry can serve for the construction of a very interesting popup mast.

a)

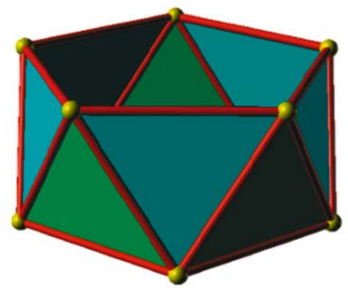

b)

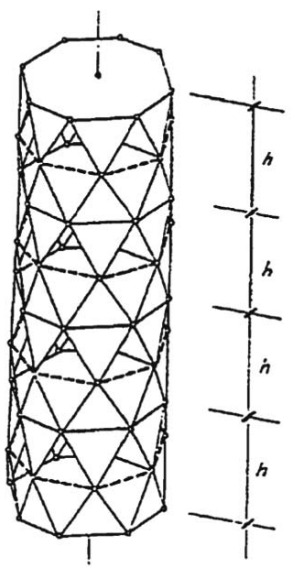

Figure 1. Regular pentagonal antiprism (a), deployable mast offered by Hegedüs [3](b)

The deployable cylindrical column offered by Hegedüs [3], shown on Fig. 1b, consists of rigid panels (octagonal panels in the figure) and rigid and elastic bars (drawn with continuous and dashed lines respectively). The mast is packed with pushing the top polygonal panel in the vertical direction. With a uniform cyclic symmetric folding the elastic bars are stretching out and the parallel polygonal panels are pushed together. It is true that the structure cannot be controlled by a single axial force [3], but with a careful symmetry control the structure can be packed to a theoretically planar truss. 
In this packed configuration the structure is in equilibrium without any external forces. This state of self-stress is not a stable position, consequently with a small perturbation the structure can snap back to the initial, deployed, strain-free configuration.
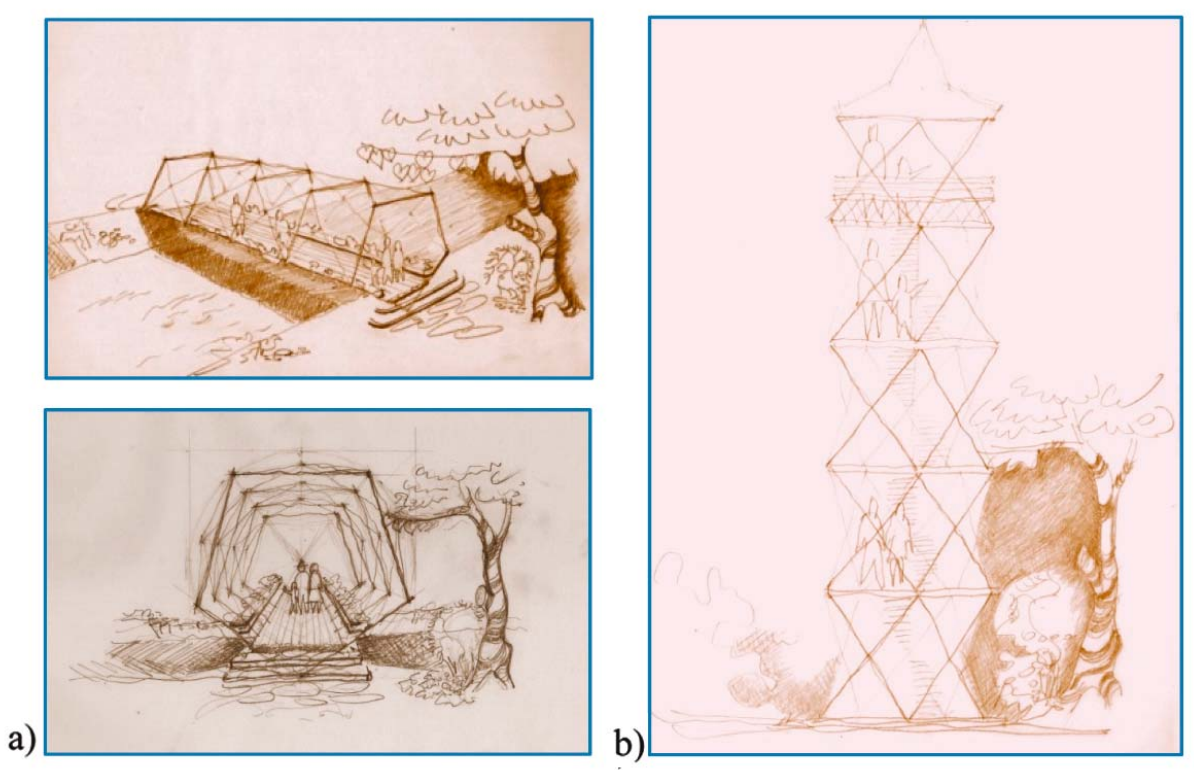

Figure 2. Application of antiprismatic deployable system to flexible architectural designs: deployable bridge (a), transportable look-up tower (b) (graphical illustration by E. Kiss)

Though the system has been already offered more than 20 years ago, no detailed analysis has been performed to investigate general mechanical behavior of the structure. Taking into account the currently prospective research field of the architectural application of dynamical and adaptive structures (e.g. [4-6]), the exploration of this immature system could be forwardlooking. This geometry could be used for a pop-out bridge construction (Fig. 2a), or as well as to look-up towers (Fig. 2b) or other adaptive designs [7-9].

The main goal of this paper is to beat a path to the design of such complex structure. In the followings the basic segment of the offered self-deploying antiprismatic column will be analyzed with analytical and numerical approaches. Though the qualitative behavior of the main characteristics of the force-displacement diagram of the basic segment was already given in [3], nevertheless, it was also found in [7] that varying the parameters of the basic segment influences largely the global packing behavior of the complex mast, namely its snapping behavior during packing. For that a profound parametric study was carried out. Besides this analysis, some approximations are also given to facilitate preliminary design of such deployable structures. 


\section{ANALYTIC INVESTIGATION}

\subsection{Kinematical equations}
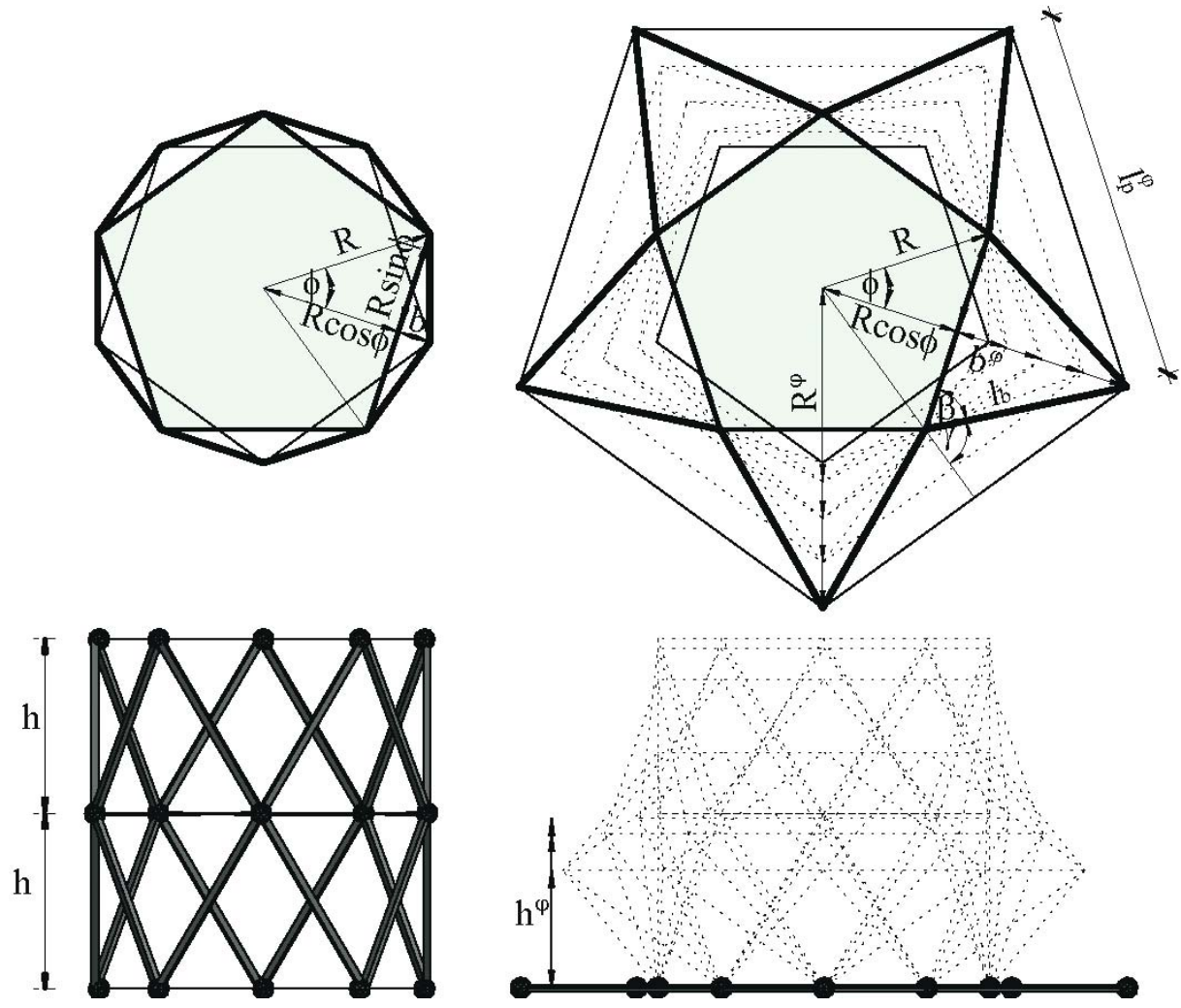

Figure 3. Planar and side view of initial (deployed) configuration (on the left) and final (packed) configuration (on the right)

Let's consider one single deployable unit of the analyzed antiprismatic truss system built from two segments of height $h$, consisting of three, initially congruent regular $n$-gons with tangential circle of radius $R$ (Fig. 3). The length of each horizontal bar in the reference, deployed state is

$$
l_{p}=2 R \sin \phi
$$

with

$$
\phi=180^{\circ} / n
$$

while the length of the rigid bracing bars is:

$$
l_{b}=\sqrt{h^{2}+R^{2} \sin ^{2} \phi+(R-R \cos \phi)^{2}}=\sqrt{h^{2}+2 R^{2}(1-\cos \phi)}
$$


The antiprismatic structure has asymmetric freedom of motions [3]. Despite this fact, from now on in the analysis only cyclic symmetrical packing will be considered. However, it is important to mention that the results deduced herein are only true in case of being able to control the movement of the structure in a way that this assumption is not violated.

During packing, the joints of the boundary polygons (the one on the top and the one on the bottom) are horizontally fixed, thus the length of their horizontal bars is constant. Furthermore, if we assume that the bracing bars are perfectly rigid (incompressible) and that the folding is uniform and has cyclic symmetry, the current length of the bars of the middle polygon stretches out during packing to:

$$
l_{p}^{\varphi}\left(R^{\varphi}\right)=2 R^{\varphi} \sin \phi
$$

where $R^{\varphi}$ is the actual radius of the tangential circle of the middle, expanding polygon which can be determined with:

$$
R^{\varphi}\left(h^{\varphi}\right)=R \cos \phi+\underbrace{\sqrt{l_{b}^{2}-\left(h^{\varphi}\right)^{2}-R^{2} \sin ^{2} \phi}}_{b^{\varphi}}=R \cos \phi+\sqrt{h^{2}-\left(h^{\varphi}\right)^{2}+R^{2}(1-\cos \phi)^{2}}
$$

The last term under square root corresponds to the length of bracing bars projected to the radius, and denoted with $b_{\varphi}$ in Fig. 3.

The stretching during packing is:

$$
\lambda\left(h^{\varphi}\right)=\frac{l_{p}^{\varphi}\left(h^{\varphi}\right)}{l_{p}}=\frac{R^{\varphi}\left(h^{\varphi}\right)}{R}=\cos \phi+\sqrt{\left(\frac{h}{R}\right)^{2}-\left(\frac{h^{\varphi}}{R}\right)^{2}+(1-\cos \phi)^{2}}
$$

In the completely packed configuration the bars of the middle polygon stretch out to the length:

$$
\left.l_{p}^{\varphi}\right|_{h^{\varphi}=0}=2 l_{b} \sin \gamma
$$

where

$$
\gamma=90^{\circ}+\phi-\underbrace{\operatorname{acos}\left(l_{p} / 2 l_{b}\right)}_{\beta}
$$

The last term refers to the angle between the fixed polygonal bars and the bracing bars (denoted with $\beta$ on Fig. 3 ) in the completely packed configuration.

Equation (7) can also be written from equation (4) and (5) by substituting $h_{\varphi}=0$ :

$$
\left.l_{p}^{\varphi}\right|_{h^{\varphi}=0}=\left.2 R^{\varphi}\right|_{h^{\varphi}=0} \sin \phi=2 R \cos \phi \sin \phi+2 \sin \phi \sqrt{h^{2}+R^{2}(1-\cos \phi)^{i}}
$$

The final stretching of the polygonal bars:

$$
\lambda_{\text {max }}=\left.\lambda\right|_{h^{\varphi}=0}=\cos \phi+\sqrt{\left(\frac{h}{R}\right)^{2}+(1-\cos \phi)^{2}}
$$


In practical design, when considering rational values of $h / R$, equation (9) can reasonably be simplified to a linear connection (Fig. 4). The value of $(1-\cos \varphi)^{2}$ is always small, and depends on the angle $\varphi$ that depends further on the number of the sides of the polygon, $\mathrm{n}$ (see equation (2)). It reaches its maximal value in the case of triangular antiprismatic segment. As for practical consideration triangular and even-sided antiprisms will be avoided, the maximum value is $(1-\cos \varphi)^{2}=0.036$, which corresponds to a pentagonal unit. Let's consider only a reasonable domain of $h / R$ :

$$
0,5 \leq \frac{h}{R} \leq 2
$$

Even in the case of the minimal value of this domain, $(h / R)^{2}$ will be one order greater than $(1-\cos \varphi)^{2}$. Accordingly, for a preliminary estimation the latter one can be neglected, which leads to a simple linear approximation (Fig. 4) of equation (9):

$$
\hat{\lambda}_{\max }=\frac{h}{R}+\cos \phi
$$

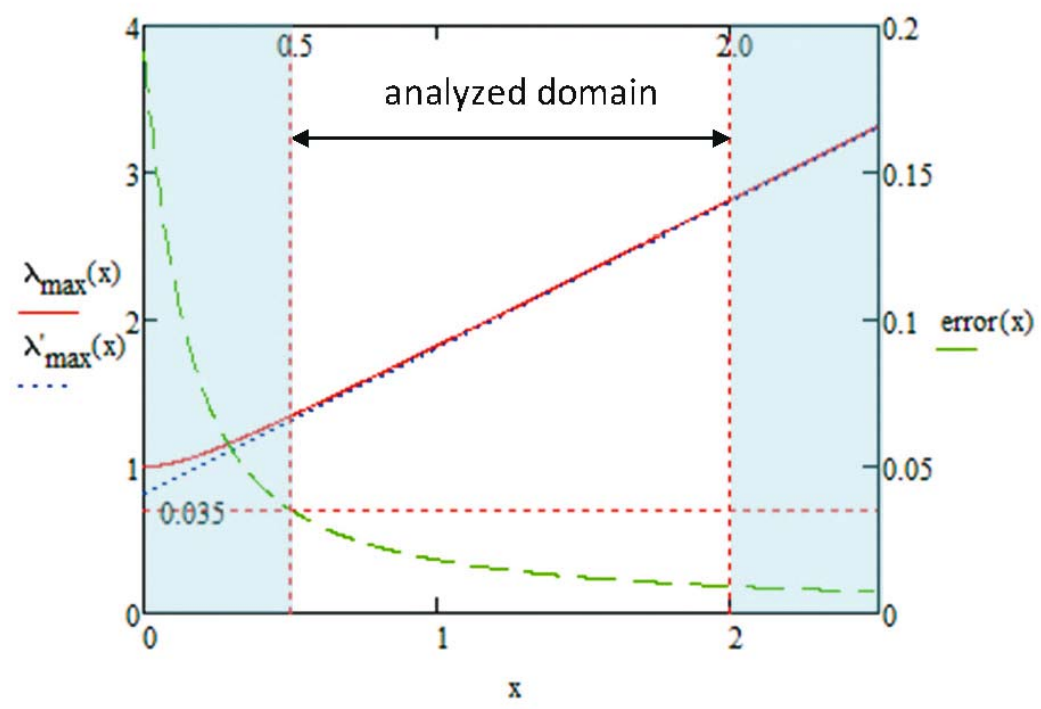

Figure 4. Linear approximation for final stretching of elastic bars $\left(\lambda_{\max }^{2}=\hat{\lambda}_{\max }\right)$ of pentagonal $(\mathrm{n}=5)$ antiprismatic unit in function of the height-radius ratio $(x=h / R)$

(exact function (equation (9)) with continuous line $\left(\lambda_{\max }(x)\right)$, approximation of stretching (equation (11)) with dotted line $\left(\lambda_{\max }(x)\right)$, error of the approximation with dashed line)

The error of this estimation can be calculated from:

$$
\operatorname{error}\left(\frac{h}{R}\right)=\lambda_{\max }-\hat{\lambda}_{\max }
$$


The maximum value of (12) is at the lower boundary of the domain (see Fig. 5), that is at $h / R=0.5$ and obviously the more sides the polygon has the less the error is (see Table 1 and Figure 5)

\begin{tabular}{|c|c|c|c|c|c|}
\hline \multicolumn{6}{|c|}{ highest error of approximation (at $h / R=0,5$ ) } \\
\hline n-gon & $\lambda_{\max }$ & $\lambda_{\max \text { approx }}$ & error $\left(\lambda_{\max }-\lambda_{\max \text { approx }}\right)$ & error $/ \lambda_{\max }$ & 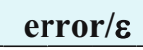 \\
\hline 5 & 1.344 & 1.309 & 0.035 & $2.62 \%$ & $10.235 \%$ \\
\hline 7 & 1.411 & 1.401 & 0.010 & $0.69 \%$ & $2.365 \%$ \\
\hline 9 & 1.443 & 1.440 & 0.004 & $0.25 \%$ & $0.817 \%$ \\
\hline 11 & 1.461 & 1.459 & 0.002 & $0.11 \%$ & $0.355 \%$ \\
\hline 13 & 1.472 & 1.471 & 0.001 & $0.06 \%$ & $0.179 \%$ \\
\hline
\end{tabular}

Table 1 Highest error of the linear approximation for final stretching of elastic bars $\left(\hat{\lambda}_{\text {max }}\right)$ for different odd sided $n$-gons

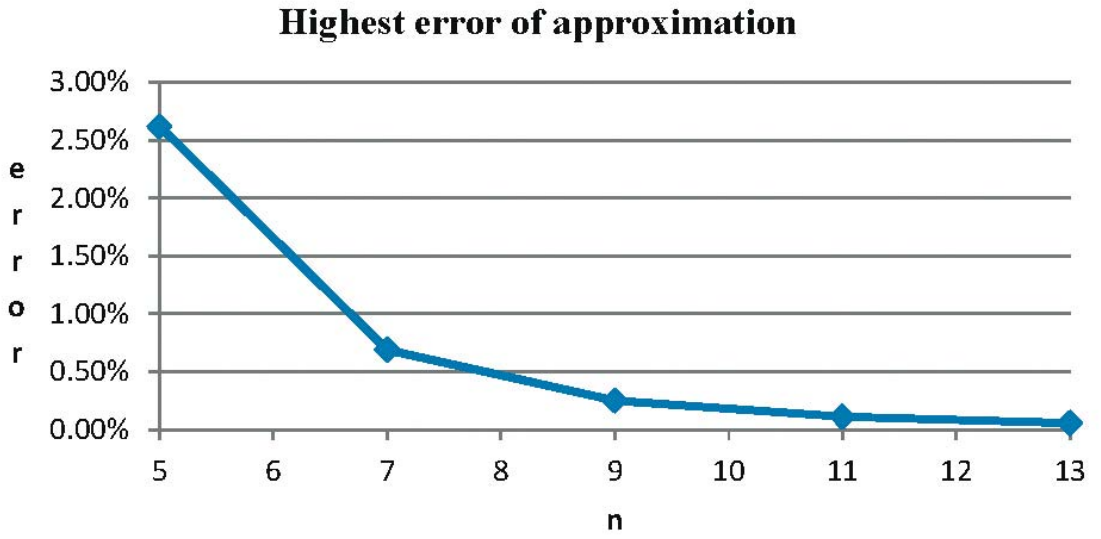

Figure 5 Highest (at $h / R=0.5$ ) error of the linear approximation for final stretching of elastic bars $\left(\hat{\lambda}_{\text {max }}\right)$ for different odd sided n-gons

This simplified linear equation can serve for the preliminary stage of design and for a good verification of results coming from the numerical analysis. Nevertheless, using the simplified equation in the case of small $h / R$ ratio (smaller than 0.5 ) the stretching is highly underestimated. This error in case of pentagonal unit reaches $10 \%$ for $h / R=0.1$ which leads to a completely fallacious result giving compression in the bars instead of stretching. 


\subsection{Equilibrium equation}
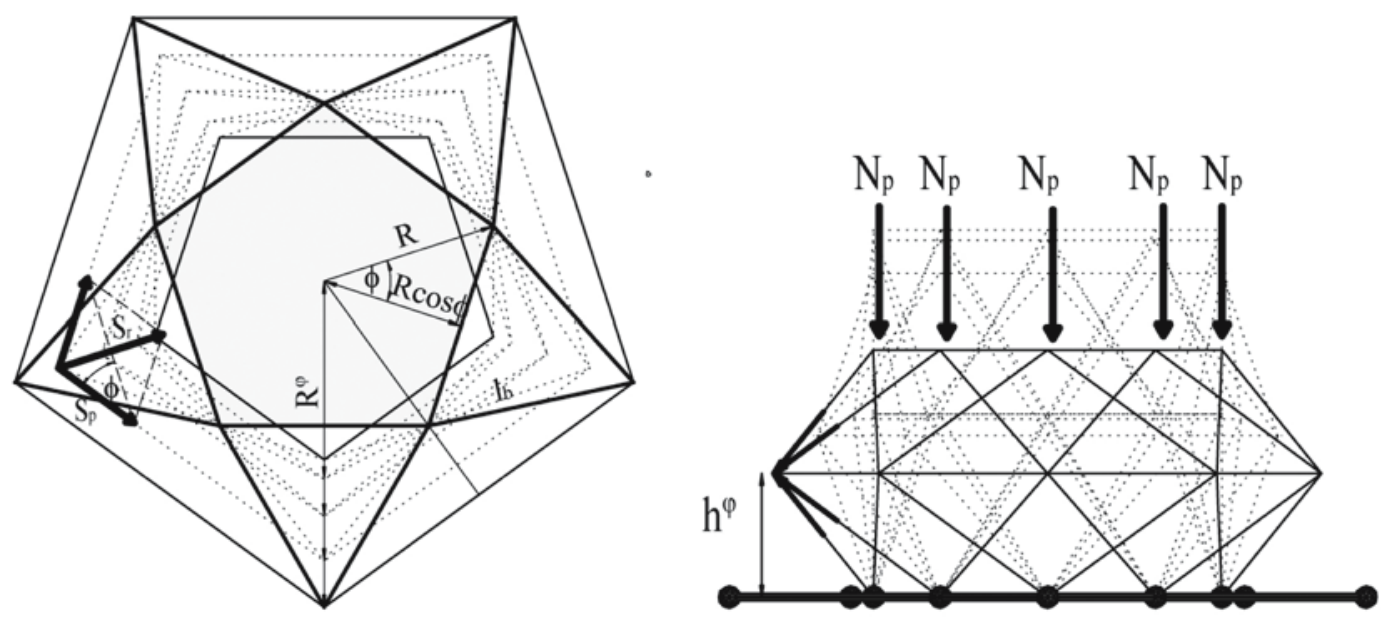

Figure 6. Equilibrium of basic deployable unit

For packing the structure, a vertical force $N$ is applied at the top horizontal polygon (Fig. 6) with a symmetrical distribution, that is, the load is $N_{p}=N / n$ at each vertex. The elastic bars of the middle polygon are tensioned by the rigid bracings coming from the top and bottom facet of the unit. With the assumption of cyclic symmetrical packing this tension force in the bars is:

$$
S_{p}\left(h^{\varphi}\right)=\underbrace{\frac{2 N}{n} \cdot \frac{R^{\varphi}\left(h^{\varphi}\right)-R \cos \phi}{h^{\varphi}}}_{S_{r}} \cdot \frac{1}{2 \sin \phi}
$$

Where $S_{r}$ denotes the horizontal radial projection of the four compression forces coming from the bracings (Fig. 6).

\subsection{Constitutive equation}

For the sake of simplicity, only the hypothesis of linear elasticity will be assumed in the analytical approach, that is, the strain in the elastic polygonal bars is:

$$
\varepsilon\left(h^{\varphi}\right)=\lambda\left(h^{\varphi}\right)-1=\frac{S_{p}\left(h^{\varphi}\right)}{E A}
$$

where $\lambda$ denotes the stretching, that is, the ratio of actual extended length and initial length of the elastic bar. 


\subsection{Equilibrium path}

Combining equilibrium (13) constitutive (14) and kinematic (5-6) equations, the forcedisplacement diagram can be written in the form:

$$
\begin{gathered}
N\left(h^{\varphi}\right)=\frac{n S_{p}\left(h^{\varphi}\right) h^{\varphi} \sin \phi}{R^{\varphi}\left(h^{\varphi}\right)-R \cos \phi}=\frac{n E A\left[\lambda\left(h^{\varphi}\right)-1\right] h^{\varphi} \sin \phi}{R^{\varphi}\left(h^{\varphi}\right)-R \cos \phi}= \\
N\left(h^{\varphi}\right)=n E A \frac{h^{\varphi}}{R} \sin \phi\left[\frac{\cos \phi-1}{\sqrt{\left(\frac{h}{R}\right)^{2}-\left(\frac{h^{\varphi}}{R}\right)^{2}+(1-\cos \phi)^{2}}}+1\right]
\end{gathered}
$$

Or in function of the displacement of the top nodes:

$$
N(u)=n E A\left(\frac{h}{R}-\frac{u}{2 R}\right) \sin \phi\left\lfloor\frac{\cos \phi-1}{\sqrt{\left(\frac{h}{R}\right)\left(\frac{u}{R}\right)-\frac{1}{4}\left(\frac{u}{R}\right)^{2}+(1-\cos \phi)^{2}}}+1\right\rfloor
$$

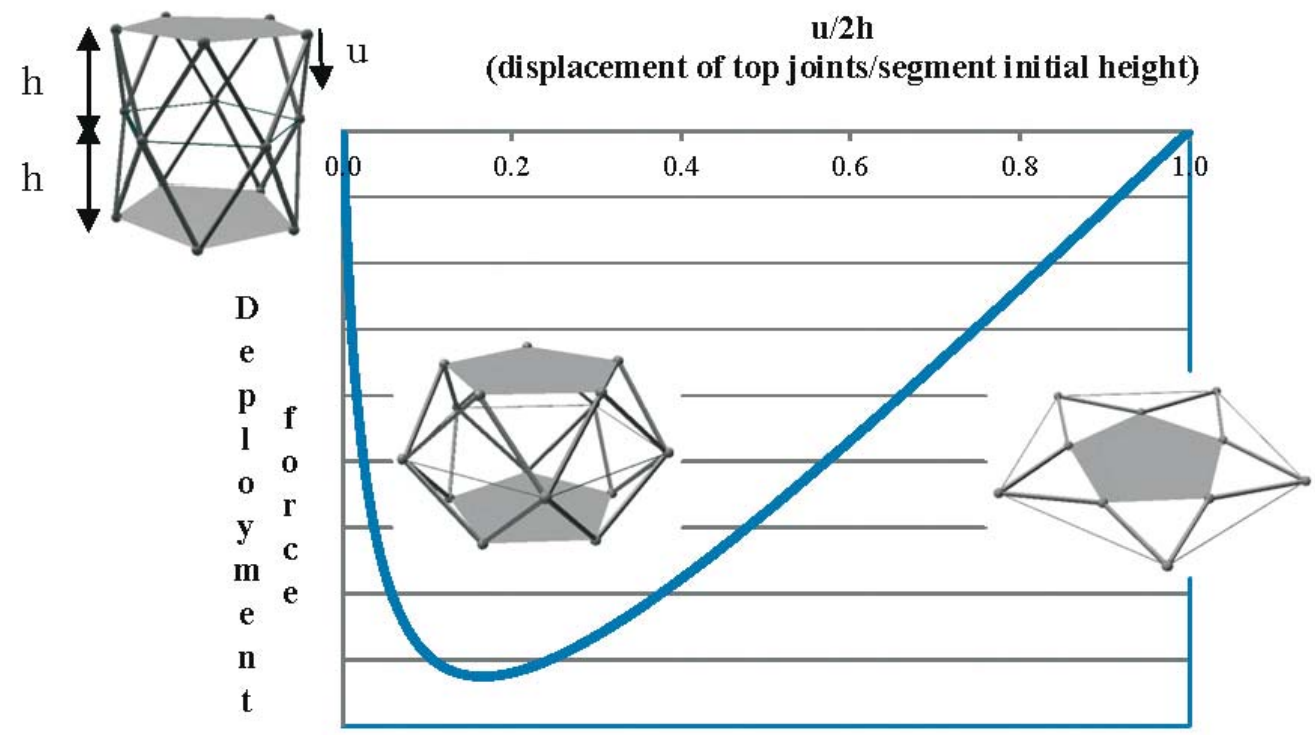

Figure 7. Equilibrium path of basic deployable unit (equation (15b))

This equilibrium path is plotted in Fig. 7, and the diagram proves that the antiprism goes through an instable phase during packing, that is, after reaching the critical force or the critical height the increment of displacement of top nodes corresponds to a decrement of force. In practice this means, if no displacement control is carried out, after the critical force, the structure snaps to the final packed configuration. 
The force-displacement diagram of the three dimensional deployable unit could theoretically be continued if the top segment is pushed further and the structure ends up in a reversed, upside down version of its initial geometrical configuration which corresponds to zero force and is stable. This phenomenon is to be avoided for the analysis of complex structures because of the failure of complete packing. However, this kind of intersection of the elements during packing in practice is not really possible. Nonetheless another freedom of motion appears at the completely packed configuration. The folded bracings can flip together up [3] allowing the lengthened elastic bars relaxing (Fig. 8). This freedom of motion can not only happen theoretically but also in practice (see physical models in Fig. 8), consequently, careful joint design is to be performed to avoid this possibility. Analogously to the definition provided at the analysis of planar structures, these two phenomena (continuing in a reversed configuration and the flipping up of the bracings) will be furthermore called 'post-packed phenomena'.
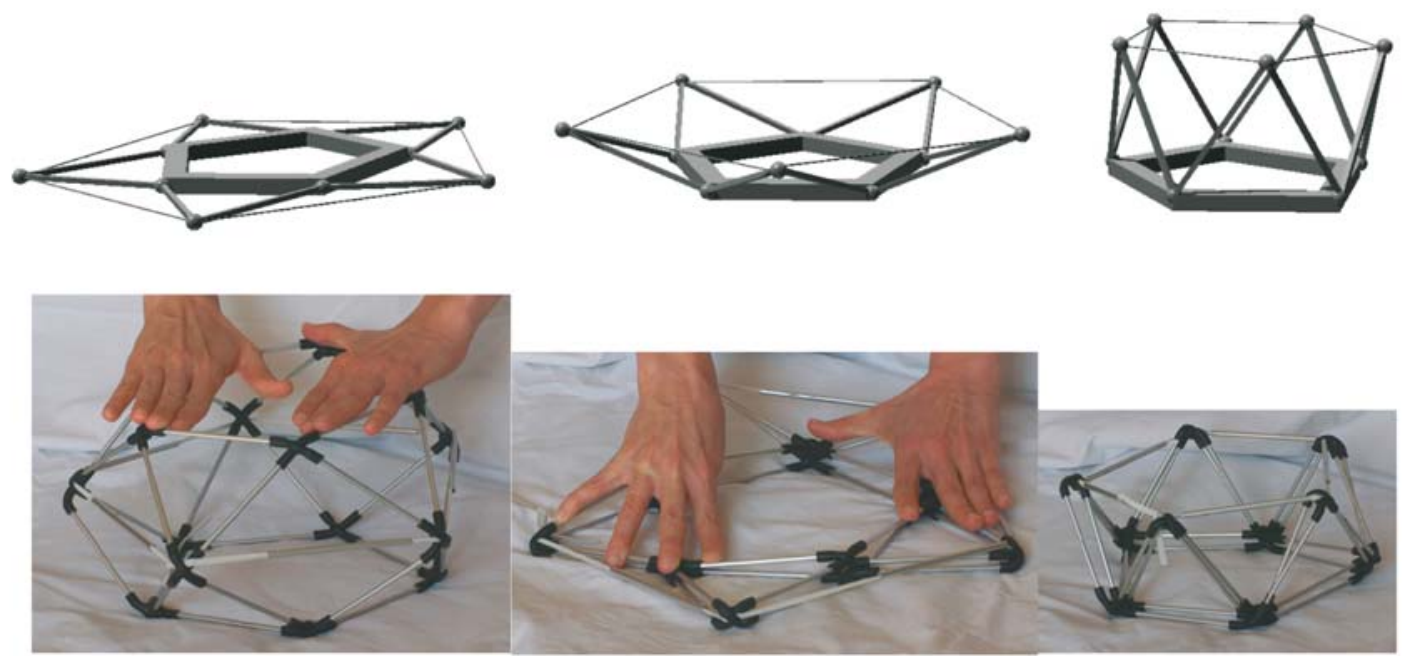

Figure 8. Post-packed phenomenon: flipping up the bracings (digital and physical models)

\subsection{Calculation of critical state}

Assuming cyclic symmetrical folding, the 3D structure's motion can be written with a single geometrical parameter. Let that parameter be $h^{\varphi}$. The critical equilibrium state corresponds to the zero value of tangent stiffness that is:

$$
\frac{d N}{d h^{\varphi}}=0
$$

Introducing the function:

$$
g\left(h^{\varphi}\right):=\sqrt{\left(\frac{h}{R}\right)^{2}-\left(\frac{h^{\varphi}}{R}\right)^{2}+(1-\cos \phi)^{2}}
$$




$$
\begin{aligned}
& N\left(g\left(h^{\varphi}\right), h^{\varphi}\right)=\frac{n E A \sin \phi}{R} \cdot \frac{\left[\cos \phi+g\left(h^{\varphi}\right)-1\right] h^{\varphi}}{g\left(h^{\varphi}\right)} \\
& \frac{d N}{d h^{\varphi}}=\frac{n E A \sin \phi}{R}\left[\frac{\left[\cos \phi+g\left(h^{\varphi}\right)-1\right] g\left(h^{\varphi}\right)-[\cos \phi-1] h^{\varphi} g^{\prime}\left(h^{\varphi}\right)}{g^{2}\left(h^{\varphi}\right)}\right]
\end{aligned}
$$

where

$$
\begin{aligned}
& g^{\prime}\left(h^{\varphi}\right)=\frac{d g\left(h^{\varphi}\right)}{d h^{\varphi}}=\frac{-h^{\varphi}}{R^{2} g\left(h^{\varphi}\right)} \\
& \frac{d N}{d h^{\varphi}}=\frac{n E A \sin \phi}{R} \cdot\left\{\frac{[\cos \phi-1] g\left(h^{\varphi}\right)}{g^{2}\left(h^{\varphi}\right)}+\frac{[\cos \phi-1] h^{\varphi}}{R^{2} g^{3}\left(h^{\varphi}\right)}+1\right\}
\end{aligned}
$$

Bringing the first two fractions in the parenthesis to common denominators and substituting equation (17) in the nominator:

$$
\frac{d N}{d h^{\varphi}}=\frac{n E A \sin \phi}{R} \cdot\left\{\frac{[\cos \phi-1]\left[h^{2}+R^{2}(1-\cos \phi)^{2}\right]}{R^{2} g^{3}\left(h^{\varphi}\right)}+1\right\}
$$

Hence the critical values of $h^{\varphi}$ can be calculated from the equation:

$$
\left.\frac{d N}{d h^{\varphi}}\right|_{h_{c r}^{\varphi}}=0 \Rightarrow(\cos \phi-1)\left[\left(\frac{h}{R}\right)^{2}+(1-\cos \phi)^{2}\right]+g^{3}\left(h_{c r}^{\varphi}\right)=0
$$

Substituting equation (17) in the last expression the critical height of the structure can be calculated from:

$$
x_{c r}^{2}=\left(\frac{h_{c r}^{\varphi}}{R}\right)^{2}=x^{2}+(1-\cos \phi)^{2}-\left\{(1-\cos \phi)\left[x^{2}+(1-\cos \phi)^{2}\right]\right\}^{2 / 3}
$$

with

$$
x=h / R
$$

Similar to the case of approximating the final stretching we can assume that both $(1-\cos \phi)^{2}$ expressions can be neglected. In this case equation (21) can be simplified to:

$$
{\widehat{x_{c r}}}^{2}=\left(\frac{h}{R}\right)^{2}+(\cos \phi-1)^{2 / 3}\left(\frac{h}{R}\right)^{4 / 3}
$$

For a nicer and still rational approximation (Figure 9) of the square root of equation (21) the critical height per radius ratio can be approximated with the Taylor formula up to the first order around the point $h / R=1$ which gives the linear approximation:

$$
\overline{x_{c r}}=a+b \frac{h}{R}
$$


The constants $a$ and $b$ depend only on the angle $\phi$ that is on $n$, the number of vertices of the polygon and can be calculated from the equation (21) and its derivative:

$$
a=\left.x_{c r}\right|_{x=1}-\left.\frac{d x_{c r}}{d x}\right|_{x=1} ; \quad b=\left.\frac{d x_{c r}}{d x}\right|_{x=1}
$$

The values for constants $a$ and $b$ are listed on Table 2 and plotted on Fig. 9.

\begin{tabular}{|c|c|c|}
\hline \multicolumn{3}{|c|}{ Constants for linear approximation of critical height/radius ratio } \\
\hline n-gon & a & b \\
\hline \hline 5 & -0.1015 & 0.9362 \\
\hline 7 & -0.0711 & 0.9624 \\
\hline 9 & -0.0522 & 0.9739 \\
\hline 11 & -0.0403 & 0.9802 \\
\hline 13 & -0.0323 & 0.9843 \\
\hline 15 & -0.0267 & 0.9870 \\
\hline 17 & -0.0225 & 0.9890 \\
\hline 19 & -0.0194 & 0.9905 \\
\hline
\end{tabular}

Table 2. Constants for linear approximation of critical height/radius ratio

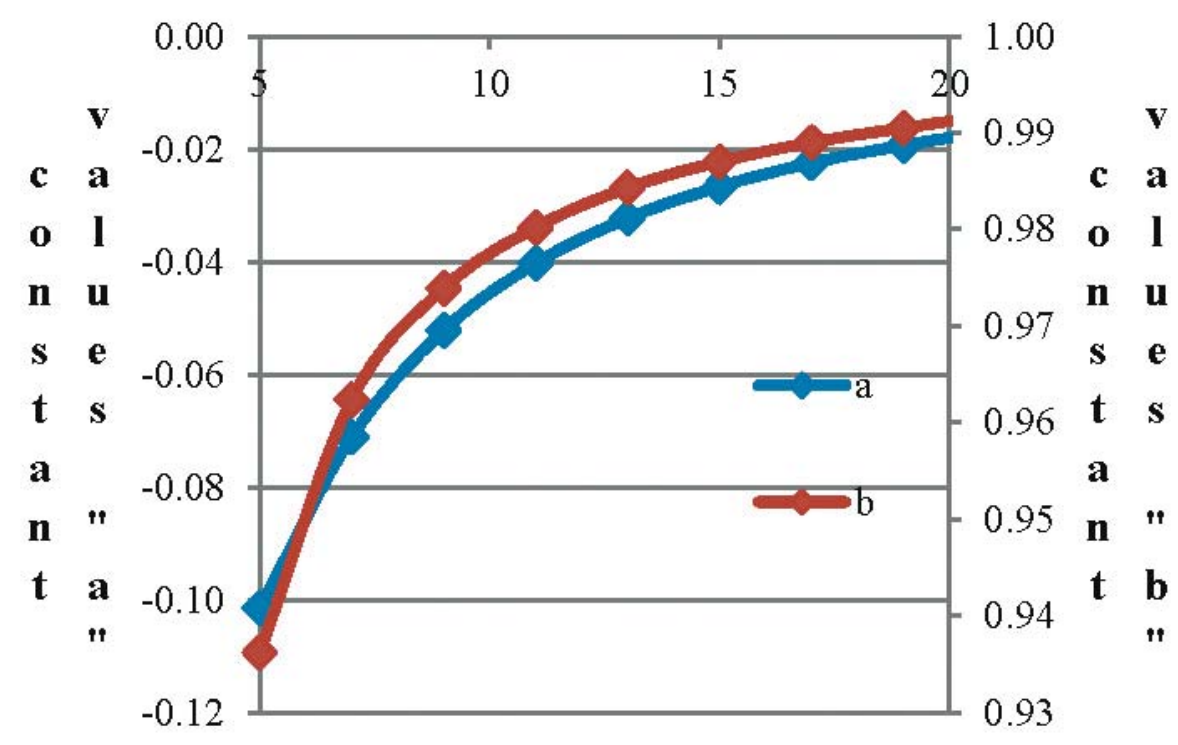

Figure 9. Constants for linear approximation of critical height/radius ratio 


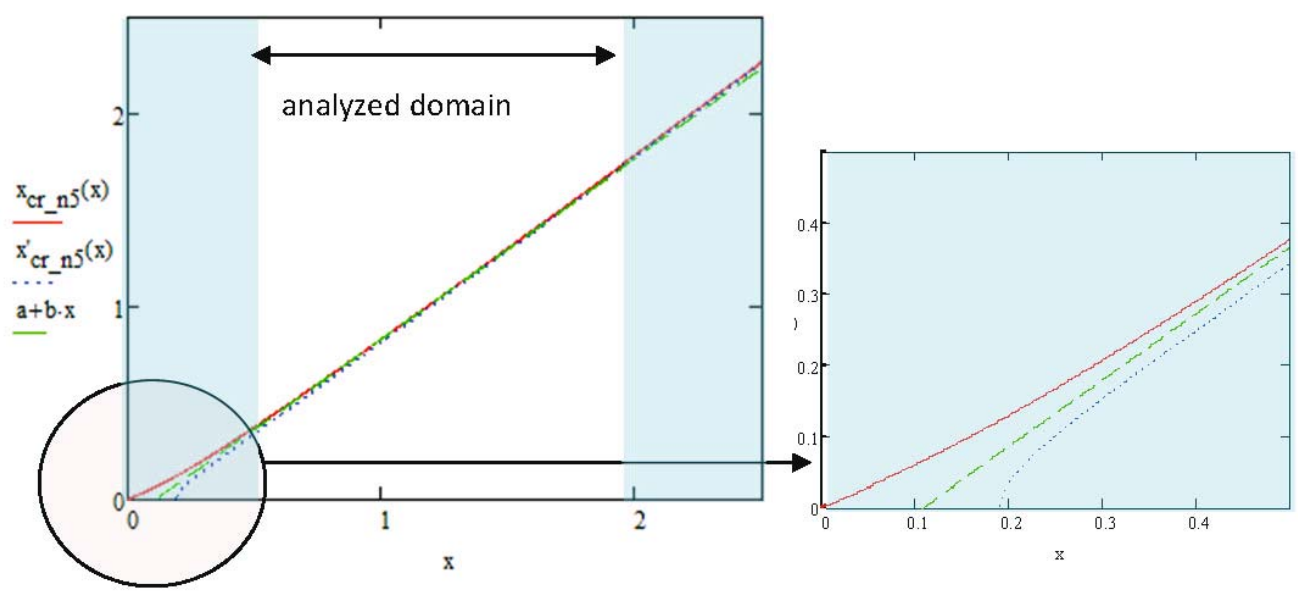

Figure 10. Approximation of critical height: critical height/critical radius $\left(x_{c r}=h_{c r} / R\right)$ in function of initial height/initial radius $(x=h / R)$, for pentagonal segment - exact solution with continuous line, approximation in accordance with equation (22) with dotted line, approximation in accordance with equation (23) with dashed line
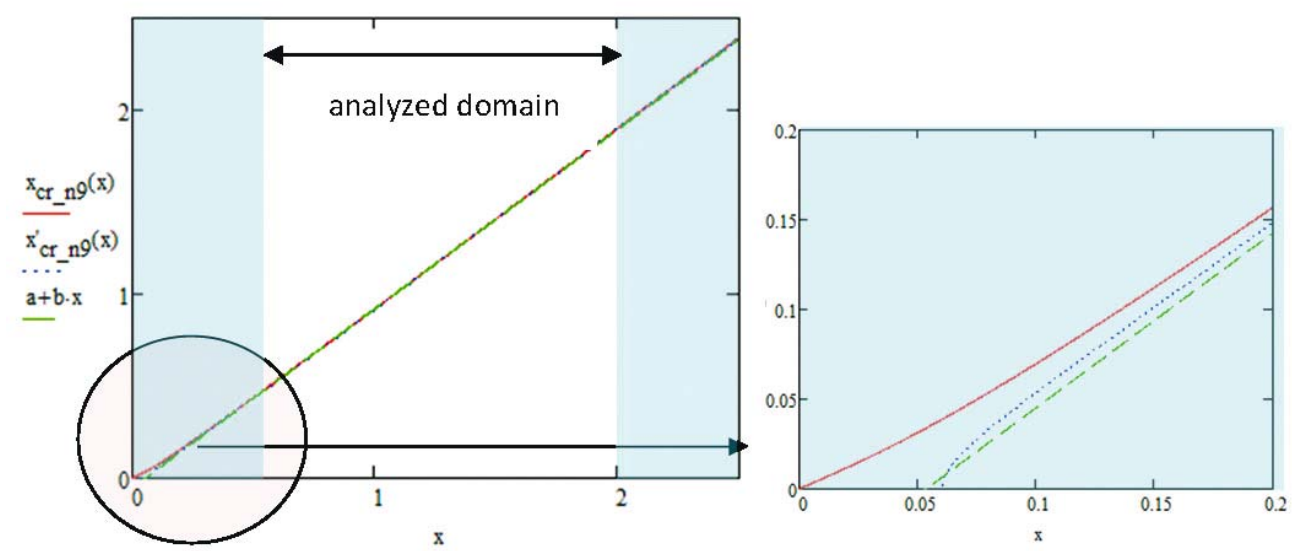

Figure 11. Approximation of critical height: critical height/critical radius $\left(x_{c r}=h_{c r} / R\right)$ in function of initial height/initial radius $(x=h / R)$, for 9-gonal segment - exact solution with continuous line, approximation in accordance with equation (22) with dotted line, approximation in accordance with equation (23) with dashed line

It can be seen from the two diagrams shown on Fig. 10-11 (plotted for a pentagonal and a 9-gonal element respectively), that depending on the actually analyzed geometry (that is, on $n$ and $h / R$ ) in some cases it is the approximation described in equation (22) and in some cases it is the one described in equation (23) that approximates better the exact value. 
Both equations underestimate the exact value. However, for domains described in (10) the offered linear equation gives an acceptable and simple preliminary estimation of the critical height.

The critical force from equations (16) (17) and (19) (21):

$$
N_{c r}(x, \phi)=n E A \sin \phi \cdot\left(\sqrt[3]{\frac{-c^{2}}{h c}}+1\right) \sqrt{h c-(c \cdot h c)^{2 / 3}}
$$

where

$$
c=(1-\cos \phi) ; h c=(x)^{2}+(1-\cos \phi)^{2}
$$

On Fig. 12 equation (25) is plotted. It can be seen that considering only the domain given in (10) the critical force can be also estimated with a linear approximation (see Fig. 12). Linearizing with the help of the Taylor formula up to the first order around the point $x=h / R=1$ :

$$
\overline{N_{c r}}=E A(d+e x)
$$

The constants $d$ and $e$ depend only on the angle $\phi$, that is, on the number of vertices of the polygon $(n)$, and can be calculated from equation (25) and its derivative:

$$
d=\left.N_{c r}\right|_{x=1}-\left.\frac{d N_{c r}}{d x}\right|_{x=1} ; \quad e=\left.\frac{d N_{c r}}{d x}\right|_{x=1}
$$

The values for constants $d$ and $e$ are listed and the error of the approximation at the boundaries of the analyzed domain are given in Table 3 and plotted on Fig. 13. To further facilitate preliminary design the critical packing force is plotted in Fig. 14 for different $n$-gonal segments.
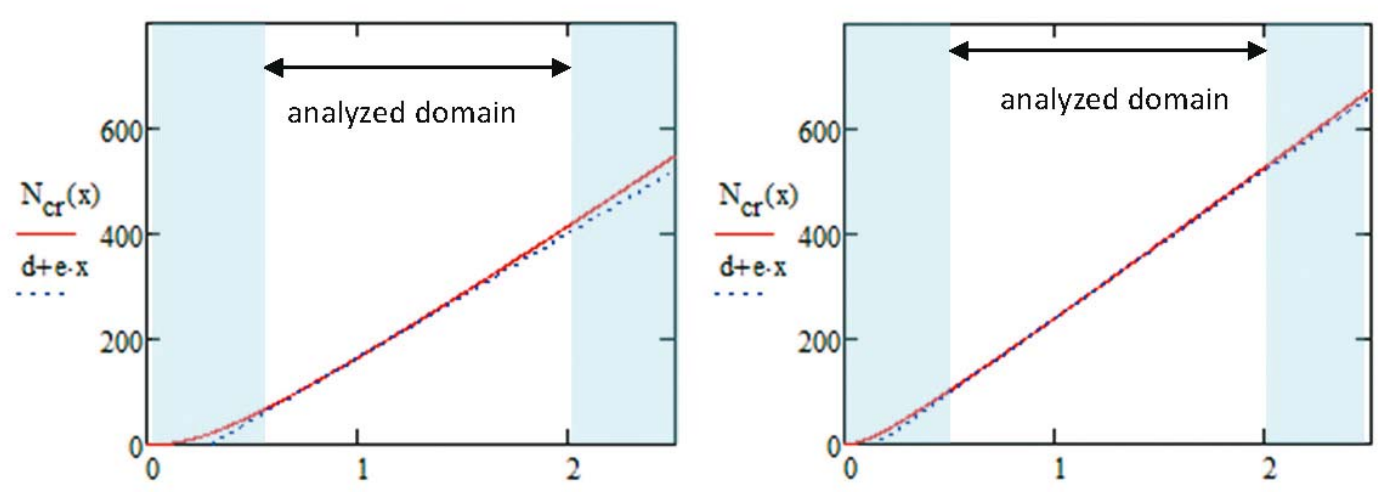

Figure 12. Approximation for the critical force: critical force $\left(N_{c r}\right)$ in function of initial height/initial radius ratio $(x=h / R)$, for pentagonal (on the left) and for 9-gonal segment (on the right) - exact solution with continuous line, linear approximation with dotted line $(\mathrm{EA}=100)$ 


\begin{tabular}{|c|c|c|c|c|}
\hline \multicolumn{5}{|c|}{ Constants for linear approximation of critical force } \\
\hline n-gon & d & e & error at h/R=0,5 & error at h/R=2,0 \\
\hline \hline 5 & -0.71760 & 2.36700 & $15.45 \%$ & $3.44 \%$ \\
\hline 7 & -0.55125 & 2.68066 & $5.91 \%$ & $1.74 \%$ \\
\hline 9 & -0.42549 & 2.82679 & $3.26 \%$ & $1.10 \%$ \\
\hline 11 & -0.33863 & 2.90828 & $2.15 \%$ & $0.79 \%$ \\
\hline 13 & -0.27736 & 2.95922 & $1.57 \%$ & $0.61 \%$ \\
\hline
\end{tabular}

Table 3. Constants for linear approximation of critical force and the error of approximation at the boundaries

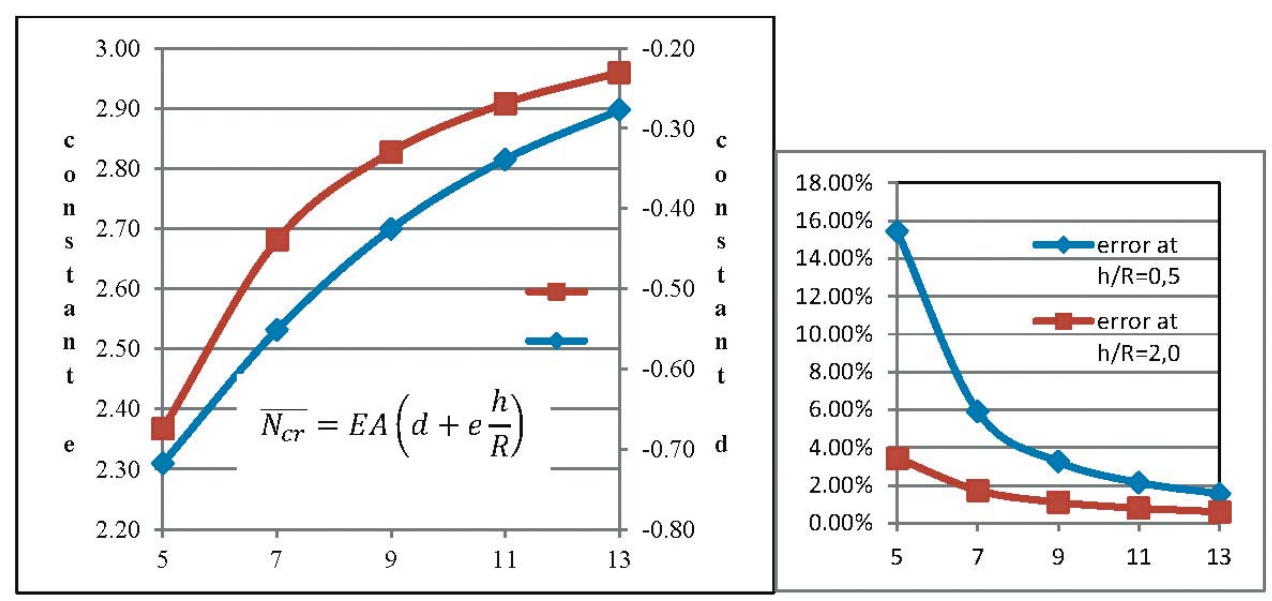

Figure 13. Constants for linear approximation of critical force and the error of approximation at the boundaries

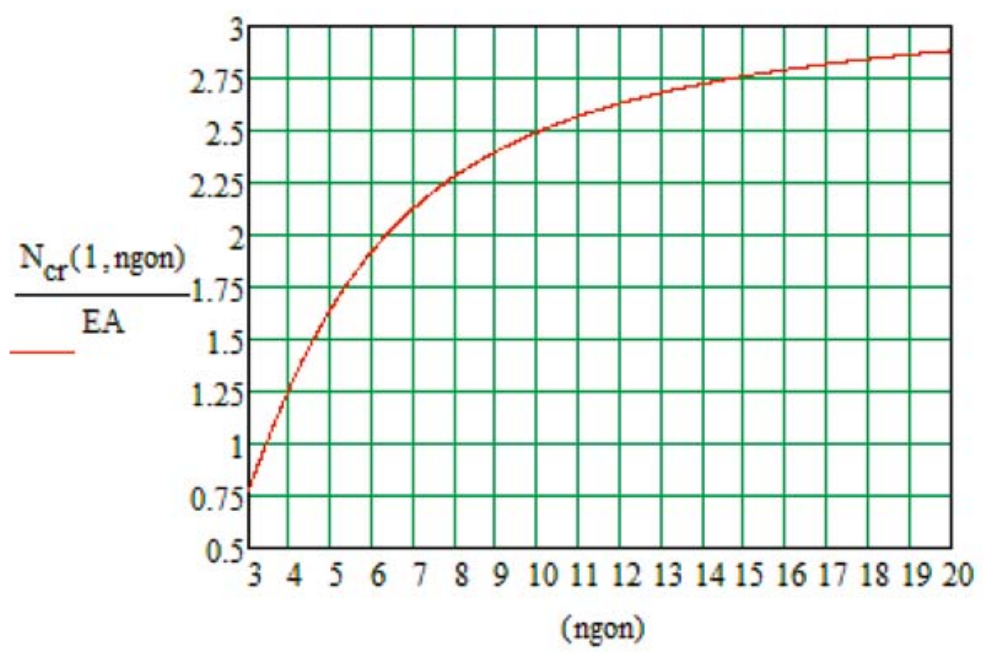

Figure 14: Critical force $\left(N_{c r}\right)$ in function of the number of vertex of the polygon for $h / R=1$ 


\section{NUMERICAL ANALYSIS}

\subsection{The numerical model}

The packing of the antiprismatic unit was simulated with non-linear finite element analysis calculated with FEAP. For the numerical model truss elements were used with constitutive model primary defined by logarithmic strain measure.

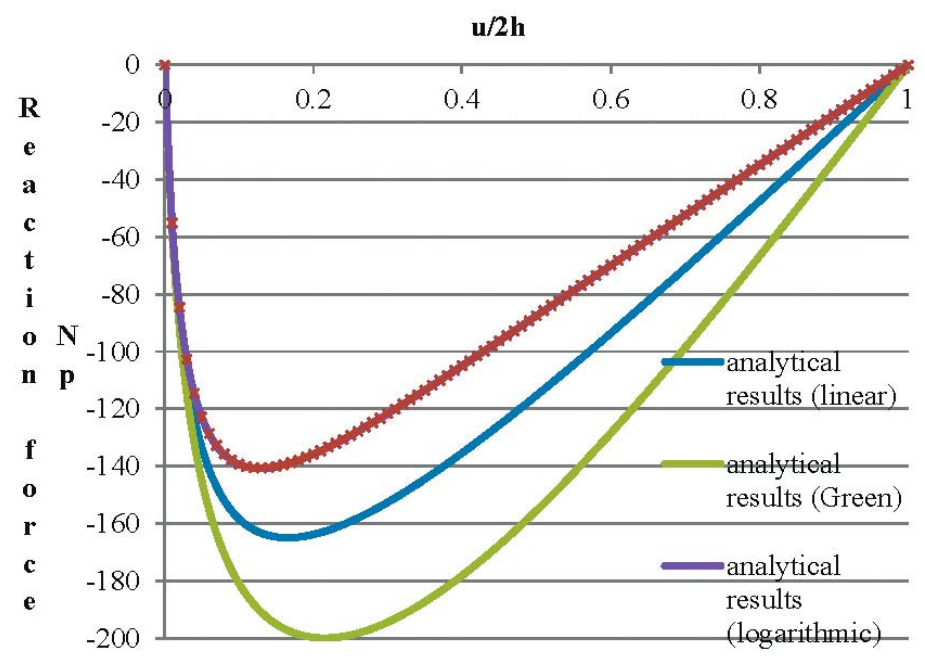

Figure 15. Comparison of different constitutive models and verifying numerical model and numerical results - force-displacement diagram of antiprismatic deployable unit (pentagonal unit, $E A=500 ; h=0.5 ; R=0.5$ )

The force-displacement diagram obtained with the numerical approach deviates from the one calculated from small strain analysis (Fig.15), especially in the larger strain domains, but the character of the force-displacement diagram is identical to analytical results. The forcedisplacement diagrams were plotted with the two different constitutive models, as well.

Assuming logarithmic strains the diagram is plotted from:

$$
N\left(h^{\varphi}\right)=\frac{n S_{p}\left(h^{\varphi}\right) h^{\varphi} \sin \phi}{R^{\varphi}\left(h^{\varphi}\right)-R \cos \phi}=\frac{n E A \ln \left[\lambda\left(h^{\varphi}\right)\right] h^{\varphi} \sin \phi}{R^{\varphi}\left(h^{\varphi}\right)-R \cos \phi}
$$

Assuming Green strains the diagram is plotted from:

$$
N\left(h^{\varphi}\right)=\frac{n E A \frac{1}{2} \lambda\left(h^{\varphi}\right)\left[\lambda^{2}\left(h^{\varphi}\right)-1\right] h^{\varphi} \sin \phi}{R^{\varphi}\left(h^{\varphi}\right)-R \cos \phi}
$$

It can be seen that the deviation is only due to the different constitutive model, the numerical errors are acceptable. The packing sequence from the simulation is plotted in Fig. 16. 


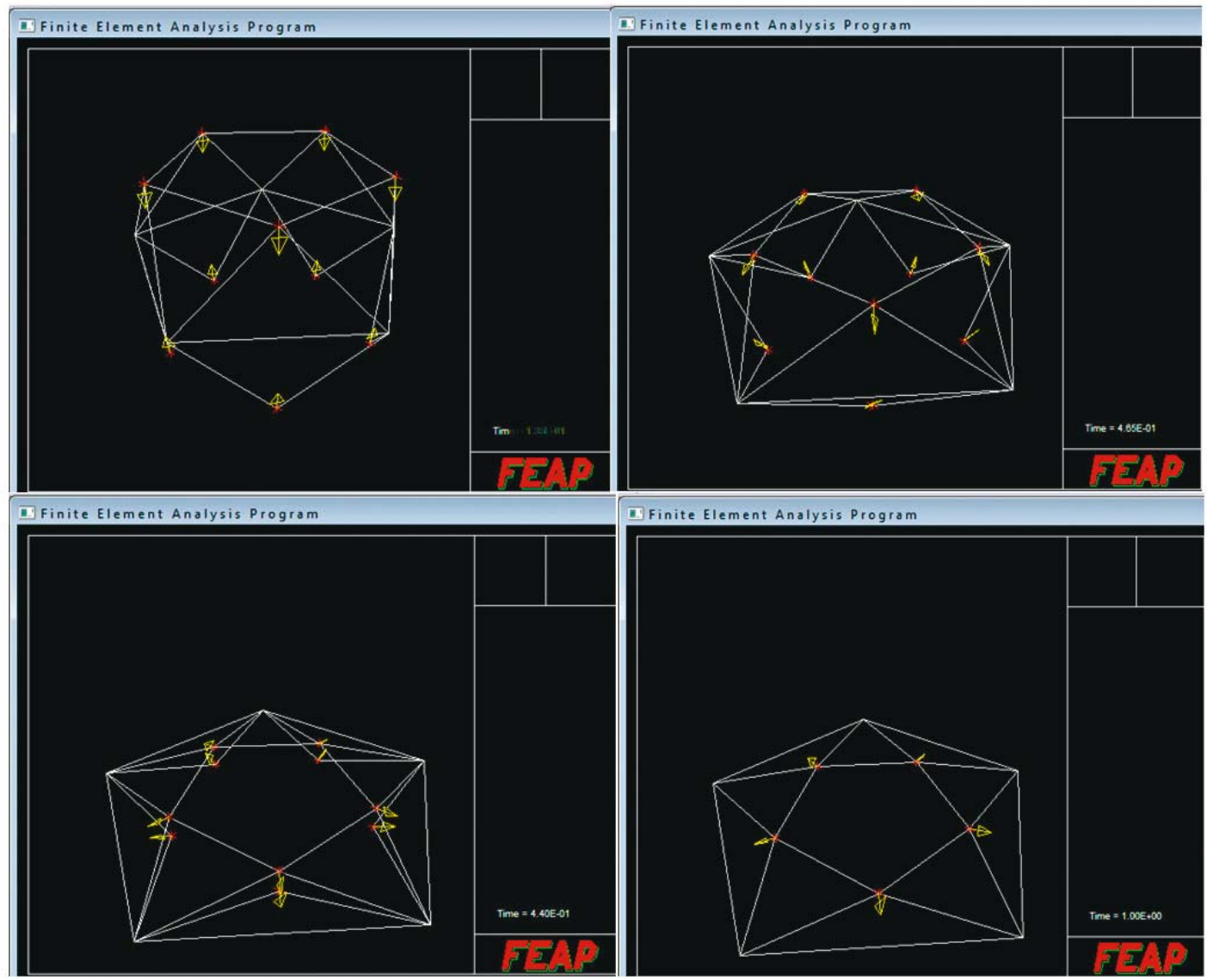

Figure 16. Packing sequence of a pentagonal basic unit

The simulation of the basic unit was carried out with displacement control; the top nodes were proportionally displaced till the bottom nodes with incremental analysis.

\subsection{Parameter analysis}

The influence of the geometrical parameters is shown on Figs. 17-20. Fig. 17 shows the forcedisplacement diagram in the case of different $n$-gonal antiprismatic basic units. It can be seen from the figure that the asymmetry of the diagram grows with increasing number of vertices. Fig. 18 is in good accordance with the analytical solution plotted in Fig. 1. Fig. 19 and 20 analyze the influence of the $h / R$ ratio on the force-displacement diagram and the packing force. From Fig. 19 it can be seen that, as the ratio grows, the asymmetry of the force-displacement diagram gets emphasized. Furthermore, Fig. 20 proves that the linear approximation deduced from analytical results (equation (27) and Fig. 12) is reliable. 


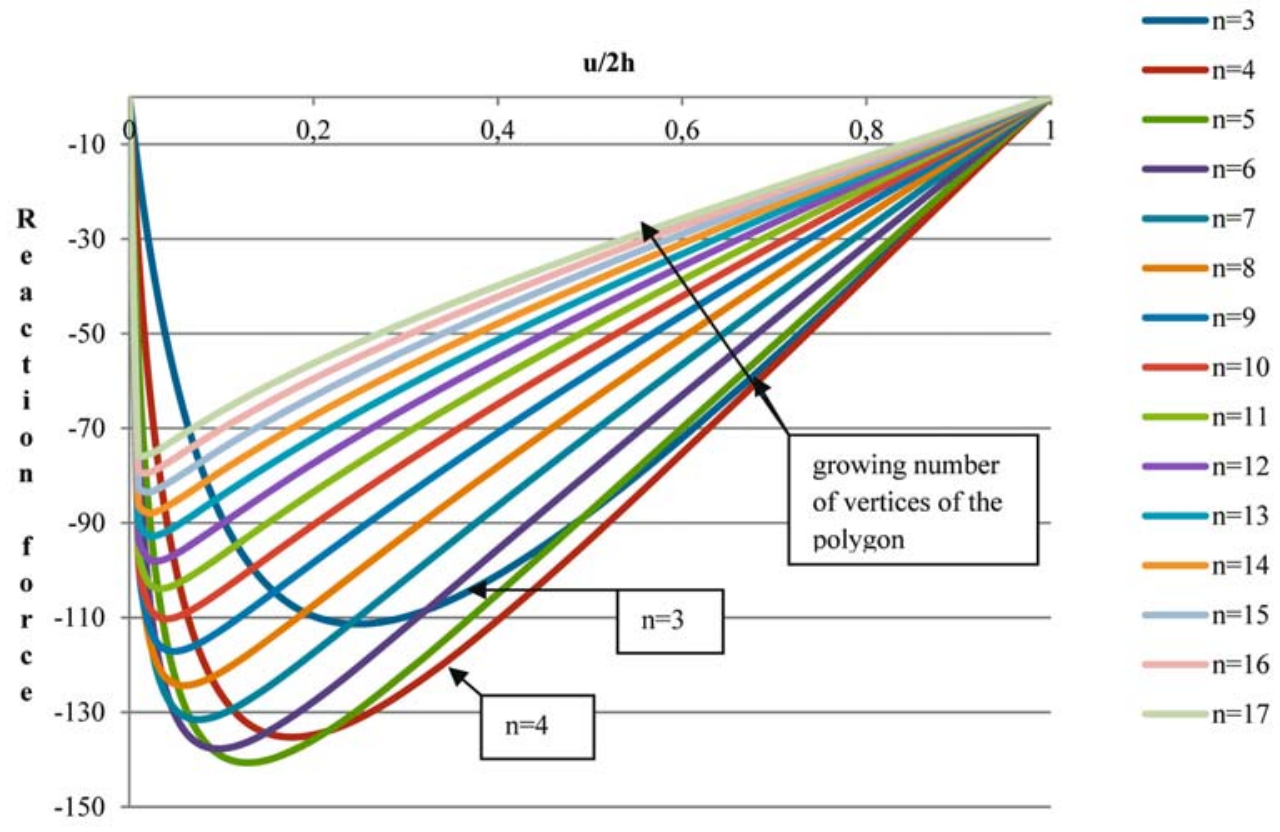

Figure 17. Influence of geometrical configuration - force-displacement diagram of basic unit in the case of different n-gons $(E A=500 ; h=0.5 ; R=0.5)$

Packing force in the case of different polygons

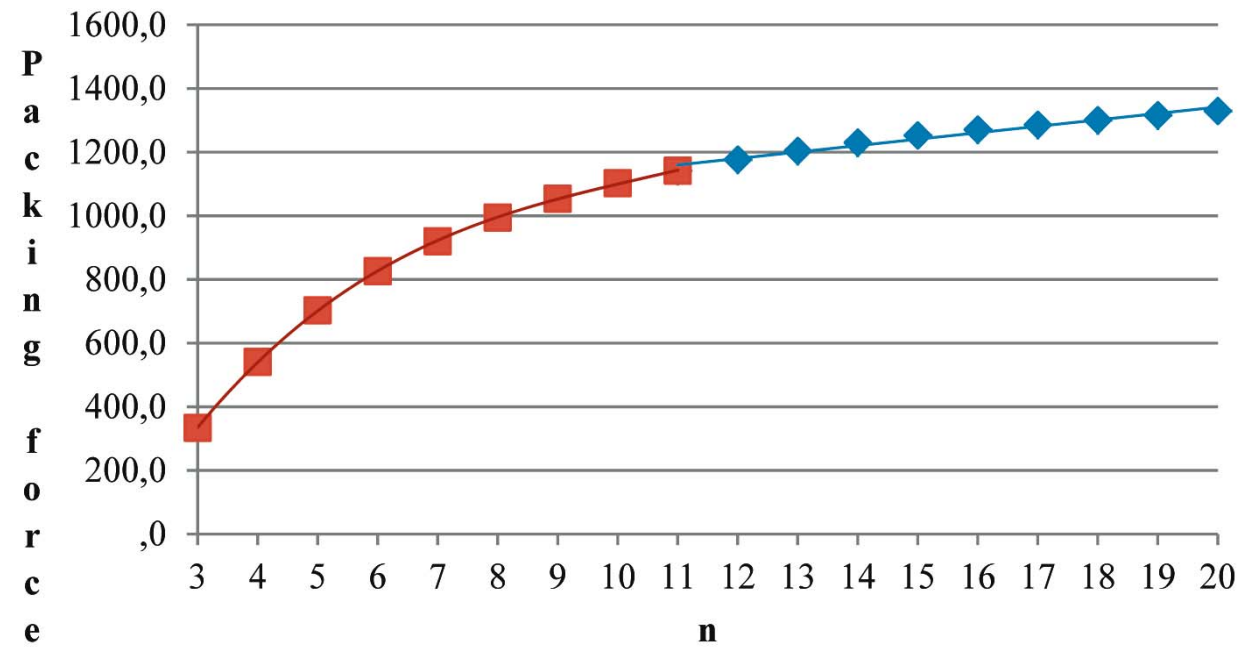

Figure 18 Influence of geometrical configuration - packing force (maximal reaction force multiplied by the number of vertices) of basic unit in case of different $n$-gons

$$
(E A=500 ; h=0.5 ; R=0.5)
$$




\section{Force-displacement diagram —influence of geometrical configuration}

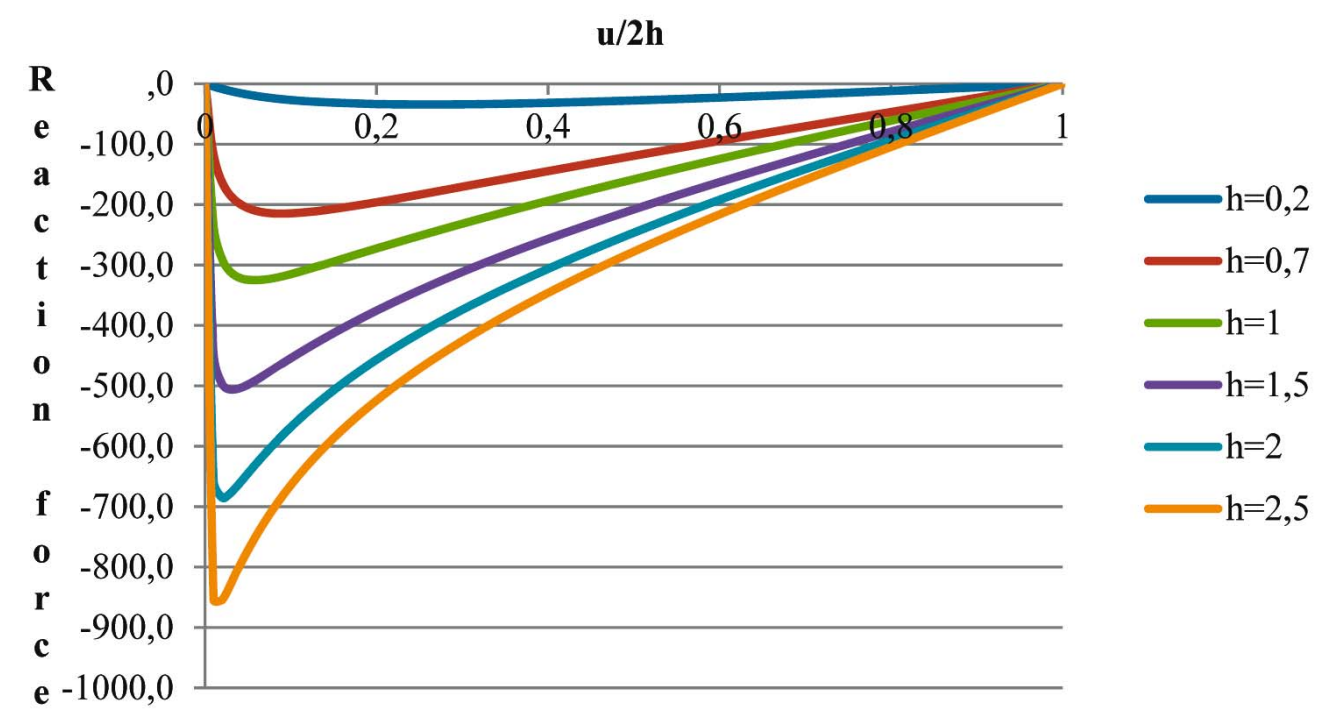

Figure 19. Influence of geometrical configuration - force displacement diagram of basic unit in case of different $h / R$ ratios

$(E A=500 ; R=0.5)$

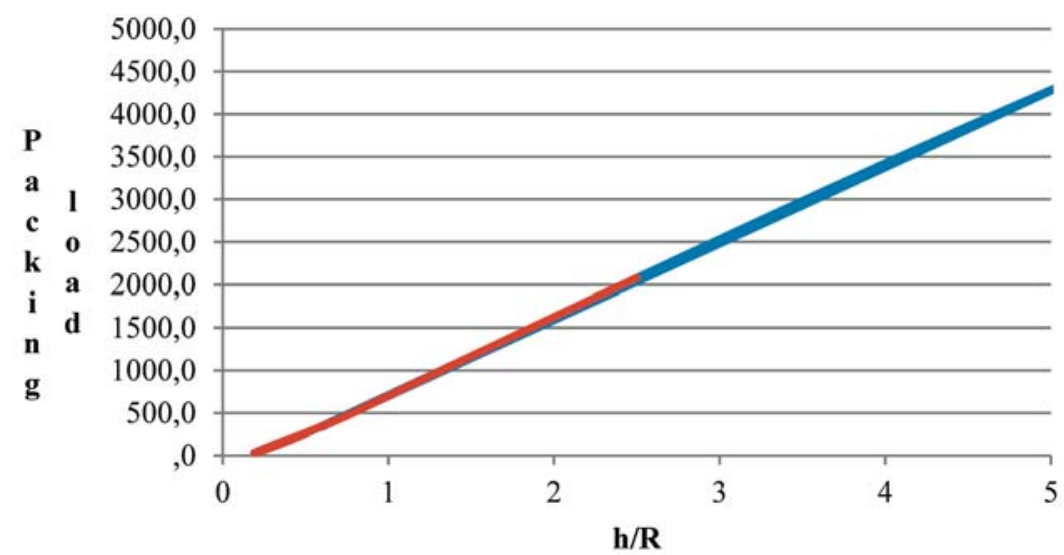

Figure 20. Influence of geometrical configuration on the packing load in the case of different $h / R$ ratios $(E A=500 ; R=0.5($ red $) R=1$ (blue $)$

\subsection{Influence of kinematical assumptions}

The above investigations were carried out with the assumption of stiffened top and bottom polygons which corresponds to the offered deployable structure of Hegedüs. If these polygons are not replaced with stiffened plates but consists of only horizontal elastic bars the structure goes to a very different equilibrium path. (Fig. 21) 
Without horizontally fixing the top nodes, the horizontal bars of the top polygon will be compressed and pushed through the middle polygon and finally by flapping up the rigid bracing bars finding back its original length with a pushed together shape.

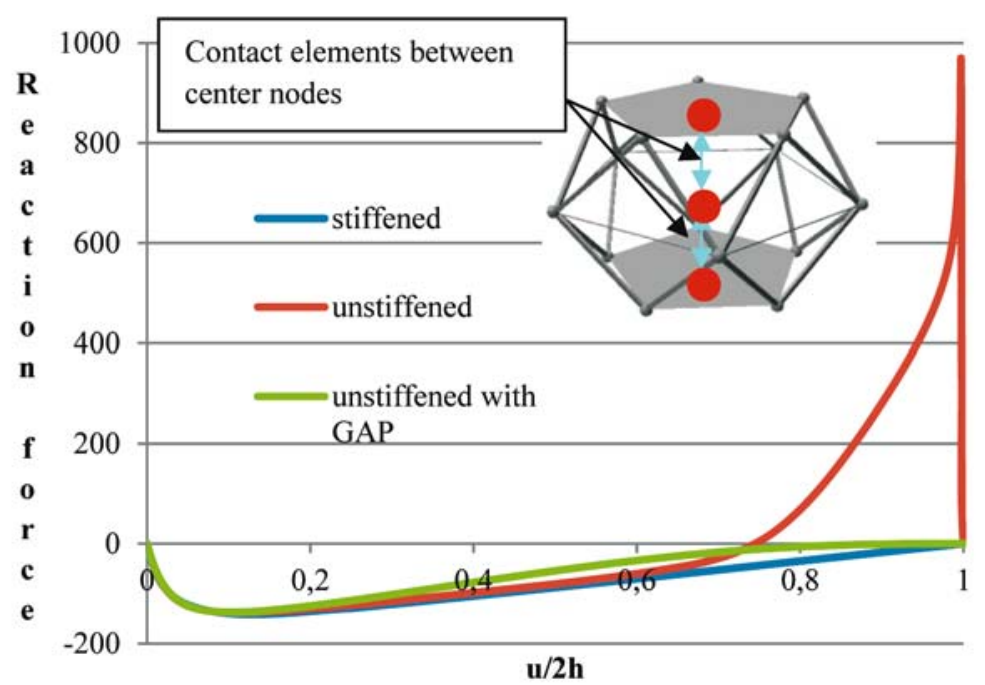

Figure 21. Infulence of changing kinematical assumption - fore-displacement diagram of non-stiffened antiprismatic unit, $(n=5, E A=500 ; h=0.5 ; R=0.5)$

To avoid this phenomenon a contact element ('gap' element) has been added to the model. A penalty approach was used to enforce a restraint between the middle and the boundary polygons. The restraint force is governed by a penalty parameter and the distance in between the center points of the polygons. When the two nodes approach each other, the contact force increases, and keeps the polygons from intersecting. The force-displacement diagram in this case is plotted with green line in Fig. 21.

\section{SUMMARY}

The basic segment of a specific system, a deployable antiprismatic lattice structure has been herein investigated. The goal of this investigation was to ground further analysis and facilitate the preliminary design of the complex system built up from these basic building blocks. This cylindrical structure, derived from the well-known Yoshimura origami pattern and proposed by Hegedüs, is characterized by its pop-up deployment due to the energy accumulated from lengthening some bars during packing. Zero deployment-load corresponds both to the fully deployed and the compact configuration, the latter being an unstable equilibrium state corresponding to the maximal internal energy.

Numerical simulation of the packing was performed and the results were confirmed by analytical investigations. 
It was shown the structure undergoes instability phenomenon during packing. The forcedisplacement diagram, the critical force and critical height of the mentioned structures were explicitly defined for linear constitutive model, and numerically analyzed for logarithmic constitutive model. For the antiprismatic pop-up system approximations were given for the following properties:

- the maximal lengthening of the bars of the middle polygon for complete packing;

- the critical segment-height;

- the critical packing force.

Numerical parameter analysis was carried out with non-linear finite element simulation to define the influence of the different geometrical and mechanical parameters on the characteristic of the force-displacement diagram by supposing logarithmical strains. The analytical results and the given approximations were confirmed with the analytical results.

It was shown that the asymmetry of the force-displacement diagram governing the intensity of the snapping of the global structure during packing depends on the number of the vertices of the polygons and on the ratio of the initial segment height and the radius of the polygons. By increasing the number of the vertices of the polygons or by increasing the ratio the asymmetry will be more significant. The critical force increases with the increment of vertices (first quadratic and then linear relationship can be observed), and linearly increases with the increment of the stiffness of the elastic bars. The critical force depends only on the height/radius ratio, but not on the size of the structure.

The paper mainly focuses on the general behavior of the basic element of the antiprismatic structure. The novelty of this paper is the determination of the exact force-displacement diagram of the basic segment of these deployable structures, enabling a profound parametric study and the formulation of approximations to its main characteristics. These characteristics of the force displacement diagram herein evaluated play an important role in the general packing behavior of the deployable mast built up from the analyzed building blocks and the given, very simple approximations facilitate the preliminary design and the verification of the numerical results.

\section{ACKNOWLEDGEMENTS}

This work is connected to the scientific program of the 'Development of quality-oriented and harmonized $\mathrm{R}+\mathrm{D}+\mathrm{I}$ strategy and functional model at BME' project. This project is supported by the New Hungary Development Plan (Project ID: TÁMOP-4.2.1/B-09/1/KMR-2010-0002). 


\section{REFERENCES}

[1] TARnal, T.: Infinitesimal and Finite Mechanisms in S. Pellegrino Deployable Structures, page 13-143, Springer Wien New York (2001)

[2] Hegedús, I.: Analysis of Lattice Single Layer Cylindrical Structures, Space Structures, vol. 2, page 87-91 (1986)

[3] Hegedús, I.: Branching of Equilibrium Paths in a Deployable Column, International Journal of Space Structures, Special Issue, vol 8, page 119-125 (1993)

[4] Friedman, N., Farkas, Gy., Ibrahimbegovic, A.: Deployable/retractable structures towards sustainable development, Pollack Periodica, ed.: Iványi, M. „Iványi A., ed. Akadémiai Kiadó, Budapest, Vol. 6. No. 2. pp. 85-97, DOI: 10.1556/ Pollack.6.2011.2.8

[5] Friedman, N., Farkas, Gy., Ibrahimbegovic, A.: Az esernyőtől az adaptív-interaktív épületekig - Nyitható-csukható rúdszerkezetek az építészetben, Magyar Építőipar, Vol. 61. No.2, ed.: Antalffy, K., Budapest, pp. 67-73. 2011.

[6] Friedman, N., Ibrahimbegovic, A.: Overview of Highly Flexible, Deployable Lattice Structures Used in Architecture and Civil Engineering Undergoing Large Displacements, YBL Journal of Built Environment, Volume 1, Issue 1. Pages 85-103, 2013

[7] Friedman, N. et al.: On the snap-back behavior of a self-deploying antiprismatic column during packing. Eng Struct (2013), http://dx.doi.org/10.1016/j.engstruct.2012.12.035

[8] Friedman, N., Farkas, Gy., Németh, O.: Környezetbarát anyagok és technológiák a tartószerkezetek építésénél (Sustainable Materials and Technologies for the Structural Construction), Építőmérnöki Kar a Kutatóegyetemért, ISBN9789633130421, (2011) 49-54.

[9] Friedman, N., Gidófalvy, K., Hegedüs, I., Farkas, Gy., Ibrahimbegovic, A.: Deployment Analysis of an Antiprismatic Snap-through Type Cylindrical Space Truss and Proposal for a Pop-up Bridge Construction, Proceeding of the IASS-IACM 2012: International Conference on Computational Mechanics for Spatial Structures, April 2-4, Sarajevo, Bosnia Herzegovina, ed. A. Ibrahimbegovic, S. Dolarevic, M. Hrasnica, M. Madzarevic and M. Zlatar, ISBN 978-9958-638-30-5, (2012) 90-92. 